\title{
Criteri di calcolo per il rivestimento termico di ugelli soggetti ad un fusso di gas ad alta temperatura (propellenti ad elevato impulso specifico) per durate relativamente lunghe $\left(^{*}\right)$
}

\author{
F. BOSMAN $(* *)$ \\ Ricevuto il 19 Febhraio 1963
}

Riasusto. - Il presente lavoro la lo scopo di fornire dei criteri pratici di progetto per il rivestimento termico di ugelli per razzi ad elevate durate di combustione.

Lo sviluppo che ha condotto alla formulazione di detti criteri ha richiesto in una prima fase lo studio della propagazione del flusso di calore attraverso una parete doppia costituita da materiali differenti (isolante e metallo); tale studio si è conchuso definendo gli elementi base che caratterizzano il fenomeno.

Successivamente si è svolta una ricerea numerica allo scopo di esplorare il campo di variabilita di tali parametri caratteristici in vista di applicare determinati materiali e per l'involucro metallico e per il rivestimento.

Tale indagine è stata efłettuata mediante calcoli eseguiti con il computatore digitale IB.II 1620 .

A questo punto si è proceduto alla soluzione numerica del problema in forma adimensionale, la soluzione è stata effettuata per un certo numero dei parametri caratteristici allo scopo di ricoprire, dando valori determinati, tutto il campo di variabilità di questi.

Queste diverse soluzioni sono state ricavate mediante calcolatrice analogica (DONNER) simulando il fenomeno della propagazione del calore.

Diagrammati questi risultati si è infine definito un criterio per ottenere rapidamente, in base alle caratteristiche dei gas, dei materiali usati e degli spessori relativi, i parametri caratteristici per i quali il calcolo già è stato eseguito.

Sono in corso delle prove sperimentali che confermano, almeno secondo i primi risultati, le conclusioni del calcolo: i migliori accordi con la teoria

(*) Nota presentata al $2^{\circ}$ Congresso Internazionale Tecnico scientifico dello spazio. Roma, 19-23 Giugno 1962.

(**) Società Bombrini Parodi-Delfino. 
-i lanno per le sezioni del convergente, in cui lipotesi hase della teoria stessa, cioe lassenza di erosione, è rispettata.

A termine della ricerca sperimentale sarà possibile fornire dei criteri di calcolo più completi, consigrlando per ogni tipo di rivestimento preso in considerazione i coeflicienti sperimentali relativi.

Sumary. - The purpose of the paper is to fumish practical criteria for the thermal easing of nozzles that are subjected to long periods of combustion. Huch research effort has been expanded in the investigation of the variability of characteristic paremeters, particularly the selection of materials and the metalling covering for the casing. This research has been aided by the use of an IBM 1620 computer. Numerical solutions in nondimensional form have been obtained for several characteristic parameters for plating. Various solutions have been found by having an analogy calculator (DONNER) simulate the phenomenon of heat proparation. Diagrammatic results provide a criteria for finding characteristic parameters rapidly. Experimental data confirm the calculated results. When this research is completed, it will be possible to fumish more complete criteria, with recommendations for relative experimental coeflicient of everv type of ansing now under consideration.

\section{Premessi.}

In Inn motore lazzo nuo dei problemi di maggior importanza is quello di evitale il riscaldamento rlell"involucero metallico oltre i limiti consentiti delle caratteristiche dei materiali nelle condizioni di larouro.

Per i motori a propellente liquirlo il problema, come ì noto, si risolve refrigerando la camera di combustione e l'ugello com uno dei propellenti prima di inviarlo alla combustione. In tal modo si può mantenere bassa la temperatura delle parti metalliche in quanto il calore frasmesso alla parete dai gas combusti viene complet.mente asportato dal fluido refrigerante. Il fenomeno della propagazione del calore rioè si manifesta nel suo aspetto di regime, e secondo queste ipotesi si eseguono di conseguenza i calcoli relativi.

Sei motori a propellente solido il problema termico, pur restambo sempre della massima importanza, ra affrontato da un punto di vista (ompletamente diverso.

Infatti per tali notori non ì possibile applicale il principio del raflechlamento, a meno di non fare assegnamento su riserve di fluido inerti dal punto di vista della combustione; quindi il calore che la parete ricere dai gas deve necessamianente accumblansi nella parete stes- 
sa, poiché il calore scambiato con l'aria esterna, per convenzione ed irlangegiamento è una quantità completamente trascurabile.

Lat temperatura della parete cresce quindi nel tempo tendendo ad un valore di regime molto alto, pari reioè alla temperatuma dei gas caldi. Tuttavia questo valore asintotico non verrà langgiunto poiché il riscaldamento si interrompe al cessare della combustione. Il problema consiste dunque nellimperdire che il massino valore deella temperatura a finc rombustione superi i liniti consentiti.

Questi liniti, per quanto si scelgano materiali cle abbiano elevate caratteristiche meceaniche ad alta temperatura, somo sempre pinttosto bassi rispetto alla temperatura dei gas, che puo essere ad es. nei propellenti compositi, dell'ordine dei 4000 oK. Questo significa che un motore a propellente solido può avele una sufficiente durata di funzionamento solo se si rallenta opportunamente il riscaldamento dellinvolucro. La fig. seguente riporta qualitativamente il guadagno di tempo di combustione oftenuto con il lallentamento suddetto.

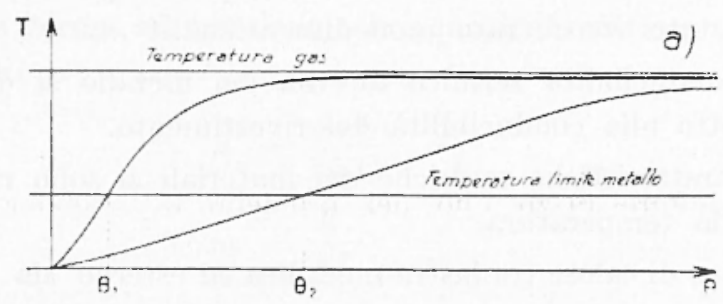

Si nota, a parita di temperatura limite laggiumgibile, la possibilitì di passales dalla durata $\theta_{1}$ alla durata $\theta_{2}$.

Dalle leggi del riscaldamento di una lastra piana che aceumuli il calore ceduto da un gas ad alta temperatura si nota come ma maggiore lentezza di riscaldamento si può oftenere farcendo assegmamento su materiali di alto "alome spereifico, di alto peso speceifico a di bassal conducibilità temical, oppure a parità di questi elementi si puó ricorrere ad elevati spessori dell'involuero. Date le aratteristiche dei materiali netallici di normale impiego, ma soluzione rera e propria non si ottiene seguendo questal stlada a meno di non ammentale ereessivamente il peso del motore usando ugedli di spessore sproporzionato.

Ena soluzione razionale si ottiene invece affidando all'involucro solo una funzione mecoanica di resistenza alle sollecitazioni di escreizio ed impedendone il riscaldannento mediante l'uso di materiali roibenti interposti tra metallo e gas caldi. Si possono, ottenere infatti in questa 
maniera degrli involucri di basso peso totale, di resistenza mecoanica adeguata, e capaci di sopportare il contatto dei gas caldi per un tempo relativamente lungo.

Si tende cioè a realizzare, mediante il concorso di più materiali, quelle caratteristiche che non è possibile riscontrare in un materiale unico, affidando ad ognumo un rompito particolane.

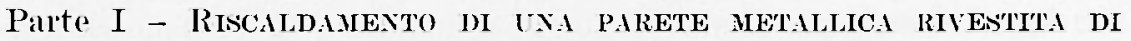
MATERIALE ISOLANTE.

In vista di determinare in sede di progetto gli elementi per la costruzione di un ugello di determinate caratteristiche, si è reso necessario uno studio generale della propagazione del calore per un involucro metallico teminiamente isolato. Io studio è stato condotto sotto le seguenti ipotesi:

1) Linvolucho si considera come mal parete piana, ('ioè il flusso di calore è stato considerato monodimensionale.

2) Isa ronducibilità termica interna del metallo si è considerata infinita rispetto alla ronducibilitì del rivestimento.

3) Ise caratteristiche termiche dei materiali si sono ritenute indipendenti dalla temperatura.

4) Il flusso di calore tra lastra metallica ed esterno sia trascurabile.

In tali ipotesi il problema si imposta nella forma seguente:

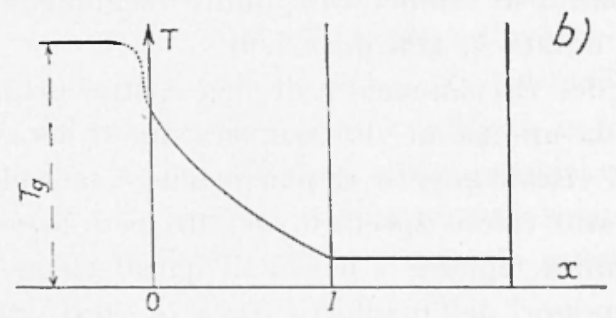

Wquazione $\frac{\partial T}{\partial \theta}=\frac{\hbar}{\partial e} \frac{\partial^{*} T}{\partial x^{2}}$

Condizioni al contorno $\left\{\begin{array}{l}X=0-X \frac{\partial T}{\partial x}=h\left(T_{y}-T\right) \\ X=l-K \frac{\partial T}{\partial x}=o_{m}\left(c_{m} l_{m} \frac{\partial T}{\partial \theta}\right.\end{array}\right.$ 
Condizioni iniziali: $0=0 \quad T=T_{o}$

ove

$T^{\prime}=$ Temperatura al tempo 0 nella ascissa $x$

$T_{g}=$ Temperatura dei gas

li: = Conducibilità temica interna della parte isolante

$h=$ Coefficiente di scambio termico dei gas

$o=$ Densità del materiale isolante

$\varrho_{m}=$ Densità del metallo

$l l_{m}=$ Spessoli rispettivamente dell'isolante e del metallo

$0-$ Tempo

La parete si è cioè schematizzata nel solo strato isolante che ricere il calore dai gas attraverso una superficie e lo cede attraverso la superficie opposta ad un serbatoio di calore (metallo).

Al fine di dare maggiore generalità al problema, e di individuare i parametri caratteristici del fenomeno in esame, si è ritenuto opportuno a mettere in forma adimensionale le equazioni scritte:

Ponendo

$$
\begin{aligned}
& n=\frac{o_{m} C_{m}}{\varrho} \frac{l_{m}}{l} \\
& m=\frac{K}{h l} \\
& \mathrm{X}=\frac{K \theta}{\underline{o} C^{2}}=\alpha \theta \\
& Y=\frac{T_{g}-T}{T_{g}-T_{o}} \\
& \xi=\frac{x}{l}
\end{aligned}
$$

Is equazioni e le relative condizioni si presentano nella forma:

Equazione

$$
\frac{\partial^{2} Y}{\partial \xi^{2}}=\frac{\partial Y}{\partial X}
$$

$$
\xi=0 \quad m \frac{\partial Y}{\partial \xi}=Y
$$

Condizioni

$$
\mid \begin{aligned}
& \xi=\mathbf{i}-\frac{\partial Y}{\partial \xi}=n \frac{\partial Y}{\partial X} \\
& \mathbf{Y}=\mathbf{0} \quad Y=1
\end{aligned}
$$


dalle quali is possibile osservare come i parametri che definiscono landamento del riscaldamento della parete (secondo le ipotesi viste) siano i tre seguenti:

$$
m, n \text { erl } a
$$

Il parametro $m$ rappresenta il rapporto delle resistenze (he si oppongono al flusso termico per unità di superficie nel passaggio dal gas alla lastra metallica, attraverso i due strati a cioc: lo strato limite del gas e la parete isolante:

$$
m=\frac{\text { Resistenza strato limite }}{\text { Resistenza parete isolante }}
$$

Il valore numerico di $m$ rappresenta perciò un indice dell’importanza che acquista un tipo di resistenza rispetto all'altra. Ad esempio quando si ha $m=1$ significa che la protezione termica fa assegmamento per metà sul rivestimento e per metà sullo strato limite; ma lastra metallica non rivestita avrà $u$ valore teorico di $m$ pari ad $\infty$; per i rivestimenti infune che si cerea di realizzale converrà proveredere ad una resistenza termica maggiore di quella offerta naturalmente dallo strato limite del gas, cioè converrà realizzare valori di $m$ inferiori ad 1.

Il parametro $n$, è il rapporto delle capacità termiche del metallo e dell'isolante riferite ad una stessa superficie. Il valore numerico di questo parametro è un indice del contributo fornito dai due strati nell'arcenmulate il calore. Per $n \gg 1$ è il metallo che funge da serbatoio termico mentre l'isolante ha solo la funzione di sbarramento termico. Per $n \simeq 1$ entrambi i materiali intervengono nella fumzione di acemmulatori, ed infine per $n \ll 1$, l'isolante assolve completamente le funzioni di sbarramento e di servatoio, mentre al metallo resta solamente quella della resistenza meceranica.

Il parametro $\alpha$, rapporto tra la diffusibilità del materiale isolante e il quadrato del suo spessore, rappresenta l'inverso del prodotto dellat resistenza termica dello strato a della sua capacità termica

$$
\frac{1}{a}=\text { Resistenza termicaa } \times \text { Capacità termicaa }
$$

E chiaro quindi che a preferibile orientarsi verso valori alti di $\frac{1}{\alpha}$ cioc verso bassi valori di $a$.

La soluzione dellequazione monodimensionale di Fourier si puo formulare in una funzione espressa come una serie di auto-funzioni. Tale funzione rappresenta la distribuzione nello strato isolante della temperatura al variare del tempo. Poiché interessa particolamente co- 
noscere il riscaldamento del metallo, la soluzione va considerata nel punto $\xi=1$ od $x=l$, dove la temperatura dell'isolante uguaglia quella del metallo.

Si ottiene cosi un'espressione della temperatura relativa variabile soltanto in funzione del tempo.

Tale esperessione, come è riportata nel lavoro degli autori John H. Grover a William H. Holter (vedi Jet Propulsion, Tol. 27, no 12 Dic. 1957) è la seguente:

$$
Y m=\sum_{i}^{\infty} \frac{2 e^{-X \lambda_{i}{ }^{2}}\left(1+m-\lambda_{i}\right)\left(1+n^{2} \lambda_{i}{ }^{2}\right) \cos \lambda_{i}}{\lambda_{i}{ }^{2}(m+n)\left\{\left(1+m^{2} \lambda_{i}{ }^{2}\right)\left(1+n-\lambda_{i}{ }^{2}\right)+n\left(1+m^{2} \lambda_{i}{ }^{2}\right)+m\left(1+n^{2} \lambda_{i}{ }^{2}\right)\right\}}
$$

Come è facile osservare la soluzione si presenta in una forma simmetrica rispetto ai parametri $m$ ed $n$, in modo cioè da non cambiare di valore sostituendoli reciprocamente. Partendo da questa constatazione gli stessi autori hamno introdotto il parametro $\mu=m+n+m \cdot n$, anche esso simmetrico. In tal modo è possibile esprimere la soluzione trovata in una nuova forma ove compare il solo parametro $\mu$ al posto della coppia $m$ ed $n$. I parametri che caratterizzano il fenomeno vengono cosi ad essere ridotti sostanziahmente a due, rioè a dire $\mu$ ed $\mu$.

L'espressione a cui ci riferisce è la seguente:

$$
Y m=\sum_{i}^{\infty} \frac{2 \mu e^{-X \lambda_{i}^{2}} \sec \lambda_{i}}{1+\mu+\mu^{2} \lambda_{i}^{2}}
$$

ove $\lambda_{i}$ sono le radici dell'equazione

$$
\lambda_{i} \operatorname{tg} \lambda_{i}=\frac{1}{\mu}
$$

\section{Parte IL - APPLICAZIONE DELAA TEORIA AI CASI PRATICI.}

a) I risultati teorici visti si presentano in forma particolamente adatta ad una applicazione pratica. Infatti traceciando nel piano $X, J$ le curve $\mu=$ cost, per vari valori di $\mu$, si ottiene un diagramma che permette il calcolo della temperatura del metallo riferita a quello del gas, dopo un tempo deteminato e relativamente a deteminati valori delle grandezze:

Spessore metallo

Spessole isolante

Tipo metallo

Tipo isolante

Coeffic. scambio termico dlei gas. 
E possibile definire lo spessore di un determinato materiale isolante in vista di mantenere la temperatura del metallo al di sotto di un prefissato limite, durante un periodo di tempo prestabilito.

Prima di tracciare le dette curve si è preferito operare una ricerca dei valori $\mu$ che si ottengono con l'impiego dei materiali metallici di impiego più comune; dei materiali isolanti attualmente usati per i rivestimenti termic $\cdot$, e dei coefficienti di scambio termico più probabili, date le caratteristiche dei propellenti usati e le condizioni di efflusso dei gas negli ugelli. La ricerca ha richiesto un lungo lavoro di calcolo come è facile intuire se si pensa al numero di variabili che intervengono nella determinazione del parametro $\mu$ ed ai numerosi materiali isolanti commercialmente reperibili. Il calcolo è stato eseguito con la calcolatrice elettronica IBM 1620. A termine di ogni calcolo la macchina ha fornito i valori di $u$ e $\alpha$ e della serie dei parametri relativi.

I materiali metallici presi in considerazione sono l'acciaio e l'alluminio, mentre per gli isolanti si è analizzato un campo notevolmente più vasto, pur limitandoci ai prodotti reperibili sul mercato specializzato; gli spessori infine sono stati scelti: per i metalli nel campo dei valori possibili date le condizioni di lavoro ammissibili, e per gli isolanti in base ai limiti consentiti dai procedimenti tecnologici.

Si sono ottenuti valori di $\mu$ variabili da 0,2 a 10 per rivestimenti in resine fenoliche rinforzate con varie sostanze inorganiche (Carbone, Amianto, Vetro ecc.), e valori superiori, cioè variabili da $4 \div 5$ fino a 300 , per i rivestimenti come i materiali del tipo Rokide (ossidi di Allumino e Zirconio).

b) Ognuna delle curve $\mu=$ cost nel piano $X, Y$ rappresenta in forma adimensionale il riscaldamento di una lastra metallica ricoperta da uno strato isolante, come già visto. Questo riscaldamento si manifesta in una forma che si presta particolarmente per una simulazione; si è così ritenuto opportuno risolvere il problema con l'aiuto di una alcolatrice analogica, anziché procedere al calcolo numerico secondo l'espressione citata.

In tal modo si è evitata la complessità del calcolo che tra l'altro implica la ricerca degli autovalori, radici dell'equazione $\lambda_{i} \operatorname{tg} \lambda_{i}=\frac{1}{\mu}$ e si è ottenuto l'andamento nella sua forma continua nel tempo anziché per punti. Il procedimento seguito per adattare il problema alla calcolatrice analogica (Donner) è stato quello di simulare la parete isolante con un determinato numero di punti riducendo il sistema, rostituito dall'equazione di Fourier e dalle condizioni relative ad un determinato 
CRITERI IJI CALCOLO PER IL RIVESTIMENTO TERMICO DI UGELLI, ECC. 273

numero di equazioni algebriche ove le incognite sono le temperature dei punti considerati.

Il valore della temperatura che ha permesso il tracciamento dei diagrammi in esame è relativa all'ultimo punto; tuttavia si sono rilevati per alcuni casi anche $\mathrm{i}$ valori di qualche punto interno onde avere un'idea dell'andamento della temperatura nell'interno dello strato.

I diagrammi della figura seguente riportano due di questi rilievi.

Per quanto riguarda la rappresentazione delle curve $\mu=$ cost nel piano $X, Y$ si è constatato che queste assumono una forma di più platicar
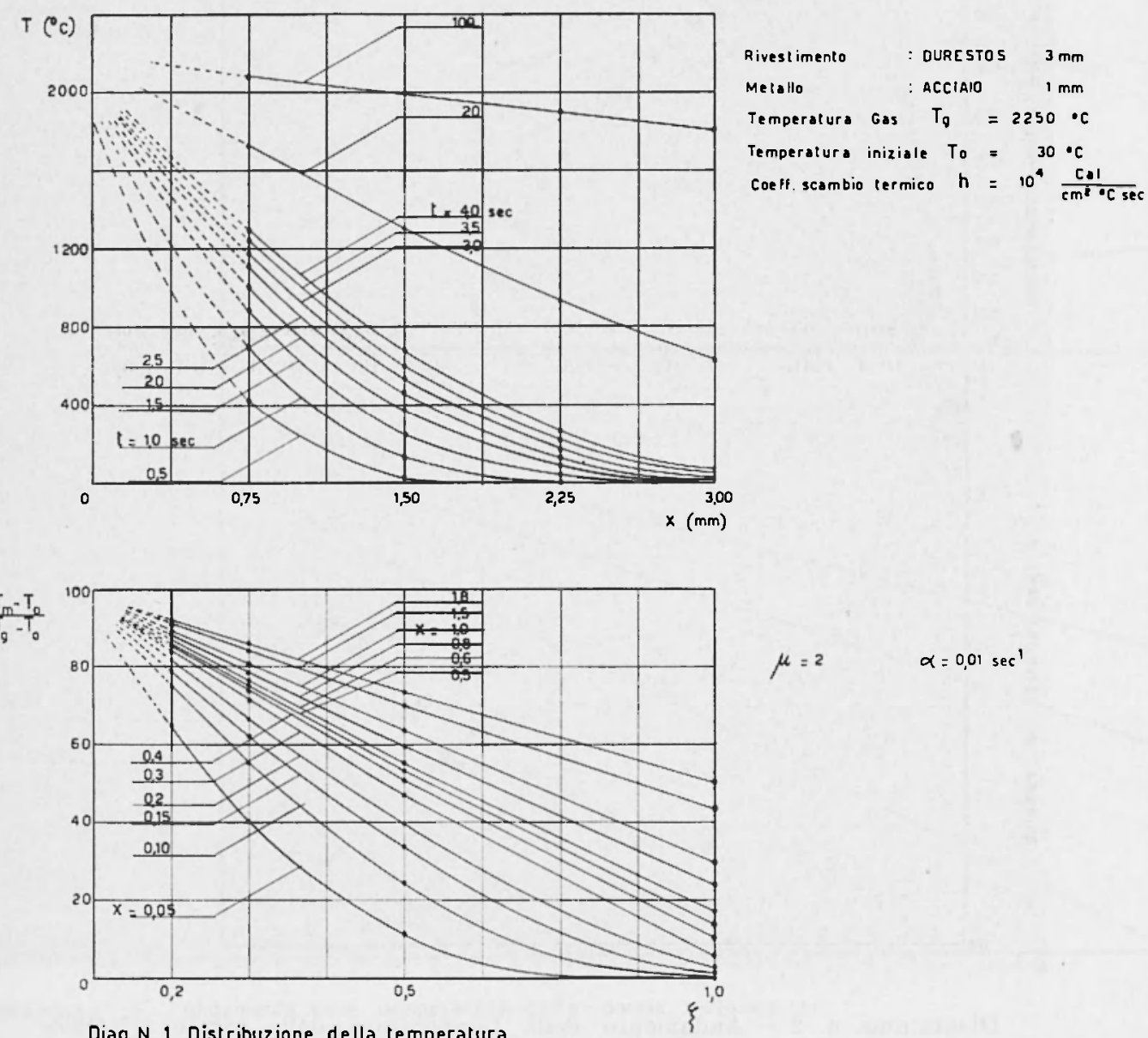

$\mu=2$

$\alpha=0,01 \sec ^{1}$

Diag. N. 1 Distribuzione della temperatura nell' interno dello strato isolante. . 
applicazione se si riportano sull'asse delle ordinate, anziché i valori di $Y$, i corrispondenti valori di $1-I=\frac{T_{m}-T_{o}}{T_{g}-\overline{T_{o}}}$.

In tal modo infatti si leggono immerlatamente le temperatures del metallo come percentuali rispetto alla temperatura dei gas.

Se inoltre si riporta aceanto al diagramma così trasformato il fascio delle rette $a=$ cost di equazione $X=\alpha 0$, tracciate nel piano $\theta, X$

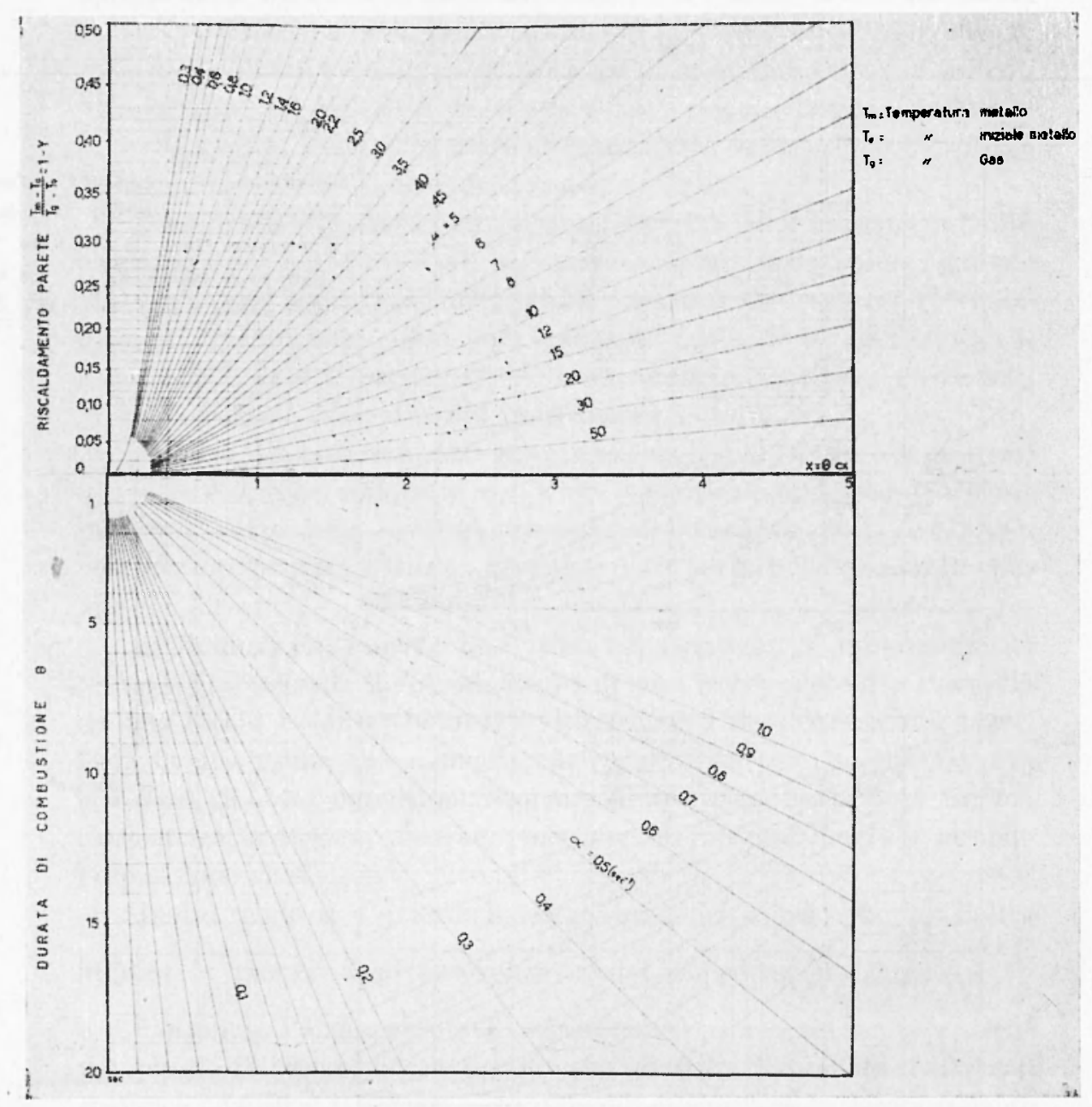

Diagramma n. 2 - Andamento della temperatura della parete metallica nel tempo per diversi valori dei parametri a e $\mu$. 
CRITERI di CALCOLO PER IL RIVESTIMENTO TERMICO DI UGELA. RCC. 275

si possono leggere i valori delle temperature relative direttamente in funzione del tempo.

I diagrammi seguenti sono stati costruiti con i criteri su esposti, ed ì chiaro come permettano la lettura del rapporto $T_{m}-T_{o} / T_{g}-T_{o}$ relativo ad un tempo 0 , per una determinata coppia di parametri $\alpha$ e $\mu$.

c) Allatto pratico il calcolo della temperatura di una parete metallica rivestita resta fin qui ancora un procedimento complesso in quanto, come già detto, la lettura nel diagramma costruito (tempera-

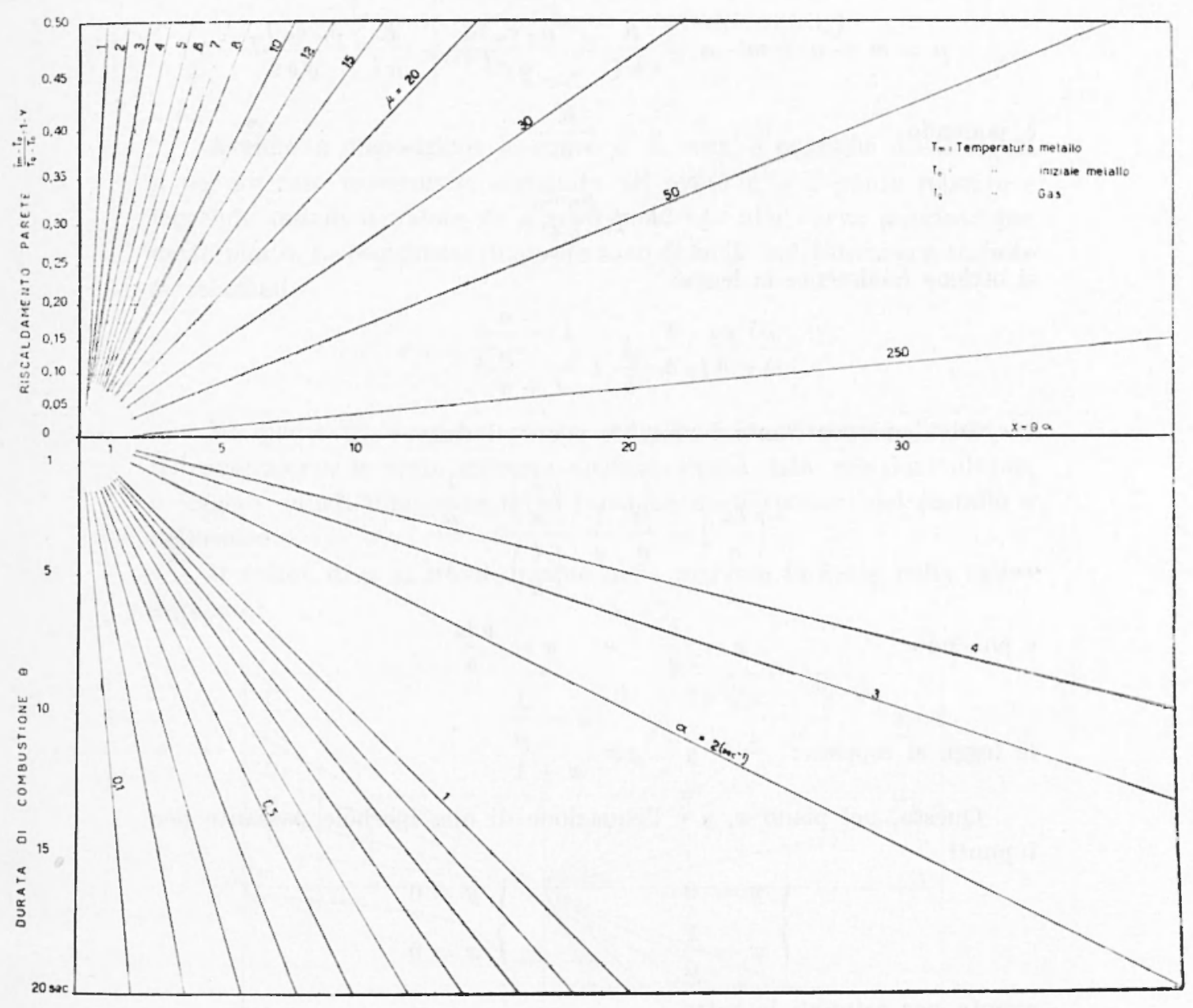

Diagramma $n^{0} 3$ - Andamento della temperatura della parete melallica nel lempo per diversi valori dei parametri $\alpha$ e $\mu$.. 
tuma-tempo) richiede la conoscenza degli elementi $\mu$ ed a che vamo determinati ogni volta in base al particolare caso in esame.

Si è reso quindi necessario ricereate un metodo pratico per una determinazione rapida di detti parametri. Per ogni accoppiamento isolante-metallo si legge in un diagramma, il quale, in base agli spessori dei materiali a del coefficiente di scambio termico dei gas, fornisce i parametri $\mu$ ed $\alpha$; con questi si può cosi entrare nel diagramma temperatulat-tempo.

Se si considera l'espressione di $\mu$ mella sua forma completa rioes

$$
\mu=m+n+m \cdot n=\frac{K}{h l}+\frac{o_{m} c_{m} l_{m}}{\varrho c l}+\frac{K}{h l} \frac{\varrho_{m} c_{m} l_{m}}{\varrho c l}
$$

a ponendo

$$
\begin{aligned}
& a=-\frac{K}{h} \\
& b=\frac{o_{m} c_{m}}{o C !}
\end{aligned}
$$

si ottiene facilmente la legge:

$$
l_{m}=\frac{\mu}{b}>\frac{l-\frac{a}{\mu}}{l+a}
$$

Divirlendo entrambi i membri per a si ricava:

$$
\left(\frac{\operatorname{lm}}{a} ; \quad \frac{\mu}{b} \frac{l}{a} \frac{\left(\frac{l}{a}\right)-\frac{1}{\mu}}{\left(\frac{l}{a}\right)+1}\right.
$$

e ponendo

$$
x=\frac{l}{a} \quad \text { a } \quad y=\frac{b l n}{a}
$$

la legge si esprime:

$$
y=\frac{x-\frac{1}{\mu}}{x+1}
$$

Questa, nel piano $x, y$ ì l'equazione di uma iperbole passante per i punti

$$
\begin{cases}y=0 & \left\{\begin{array}{l}
y=0 \\
x=\frac{1}{\mu}
\end{array}\right.\end{cases}
$$

avente per asintodi le rette

$$
x=-1
$$

ed

$$
y=\mu x-(1+\mu)
$$


CRITERI DI CAICOLO PER II, RIVESTIMENTO TERIICO DI UGELI, ECC. 277

e tangente nell'origine $y=-x$, come ì riportato in figura seguente:

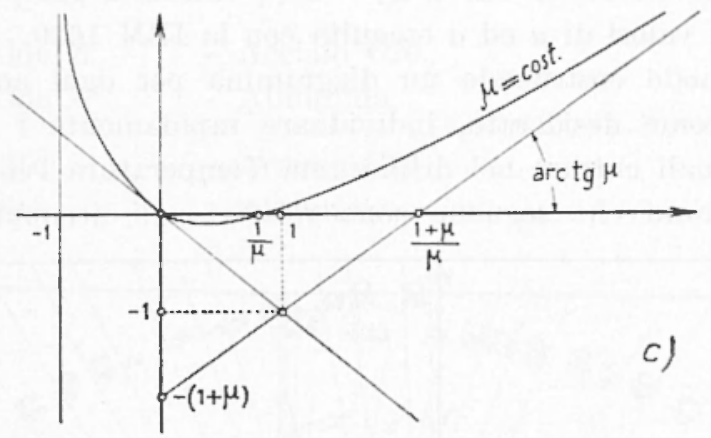

Avendo a disposizione le curve $\mu=$ cost, ̀̀ possibile il calcolo di $\mu$ per un caso particolare, segmando nel piano $x, y$ il punto relativo e leggendo quindi il valore di $\mu$ corrispondente alla curva passante per detto punto. Le coordinate di questo sono di facile individuazione tramite: le relazioni:

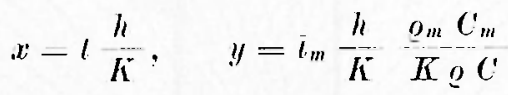

Ma per evitare anche questa complicazione si possono riportare nel diagramma le rette espresse analiticamente dalle relazioni ultime, e leggere quindi direttamente in funzione degli spessori del metallo e dell'isolante.

I.l valore di $\mu$ si trova dunque nella maniela indioata nella figura seguente:

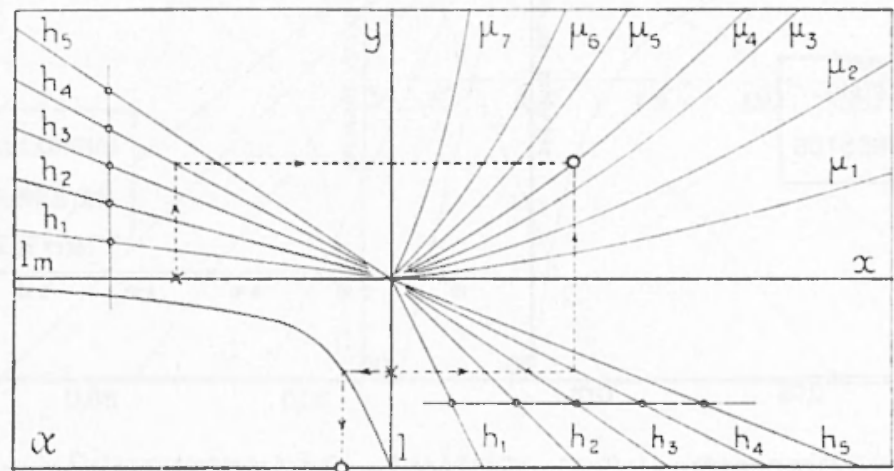


Sempre nello stesso diagramma si è riportato, utilizzando il quadrante rimasto libero, la curva $\alpha=\alpha(l)$ ealcolata già per punti nell'indagine dei valori di $\mu$ ed $\alpha$ eseguita con la IBM 1620.

In tal modo costruendo un diagramma per ogni accoppiamento si possono, come desiderato, individuare rapidamente i parametri $\mu$ ed $\alpha$ con i quali entrare nel diagramma Temperatura-Tempo.

I diagrammi che seguono sono relativi agli accopplamenti

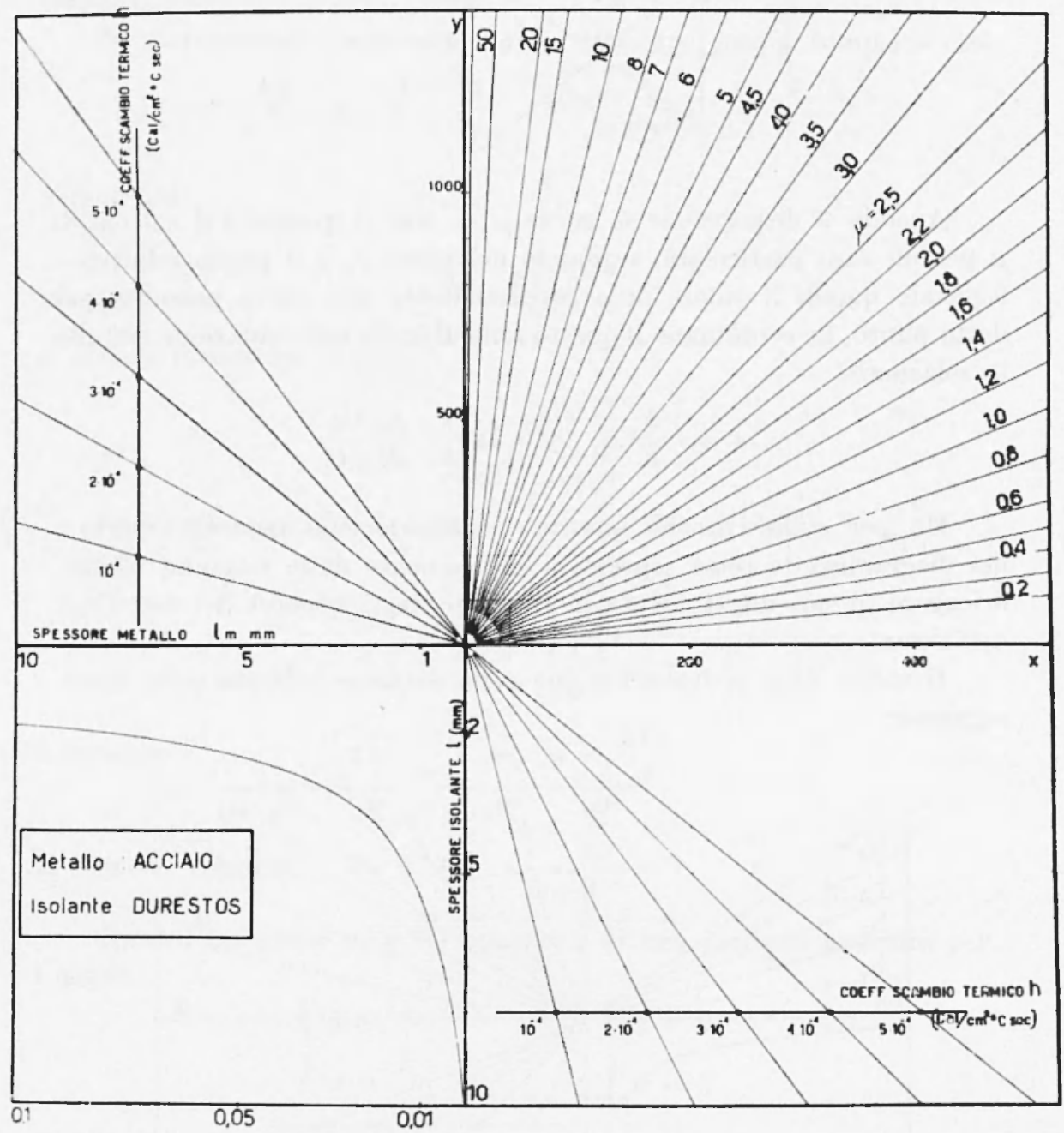

a isec')

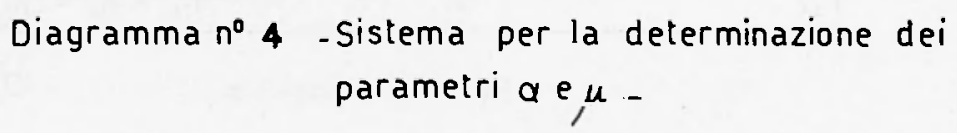


CRITERI DI CALCOLO PER IL, RIVLSTIMENTO TERMICO TI UGELII, ECC. 279

Durestos RA 9 - Acciaio C 20

Durestos RA 9 - Alluminio

Rokide $7 / \quad$ - Aceciaio C 20

Rokide $Z \quad-$ Alluminio

Glafite $\quad-$ Aceriaio C20

Grafite - Alluminio

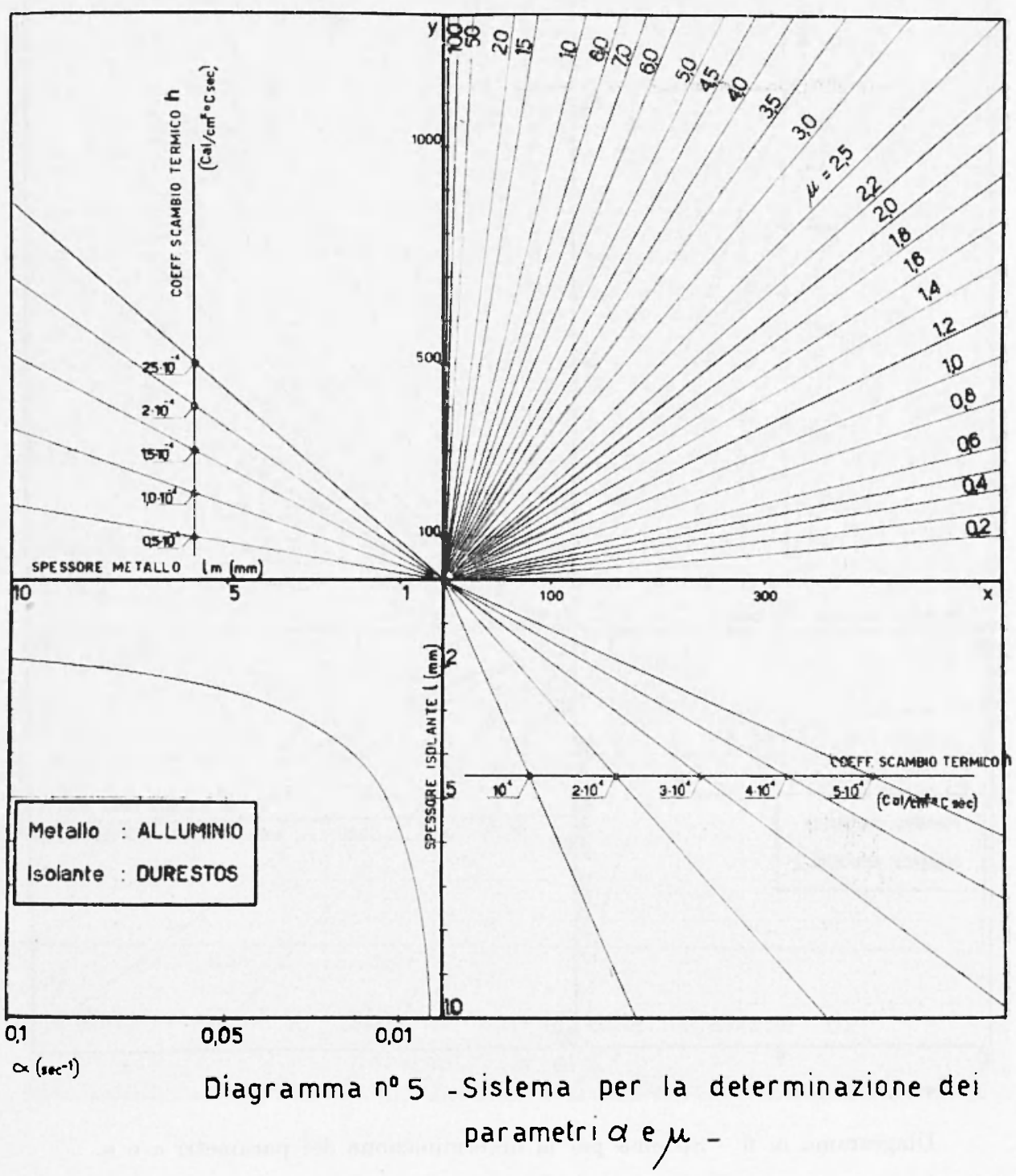


I ralori numerici delle canatteristiche dei materiali ai quali si riferiscono $i$ diagrammi costruiti sono state assunte dai cataloghi delle società produttrici a dal testo "Missile Engineering IIandbook".

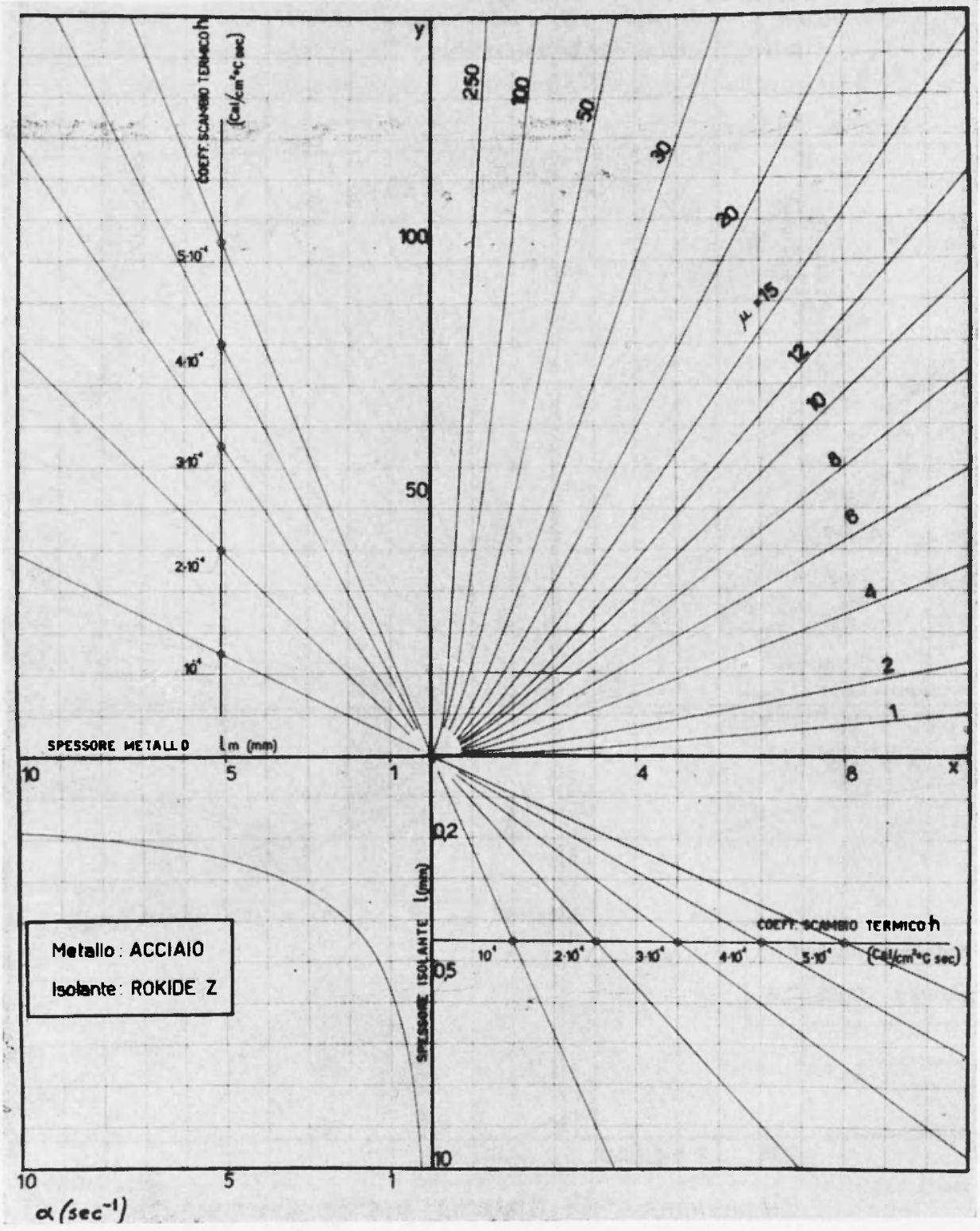

Diagramma n. 6 - Sistema per la determinazione dei parametri a e $/$. 
CRITERI di CALCOLO PER IL RIVEstimento TERMiCo di UgeLli, LCC. 281

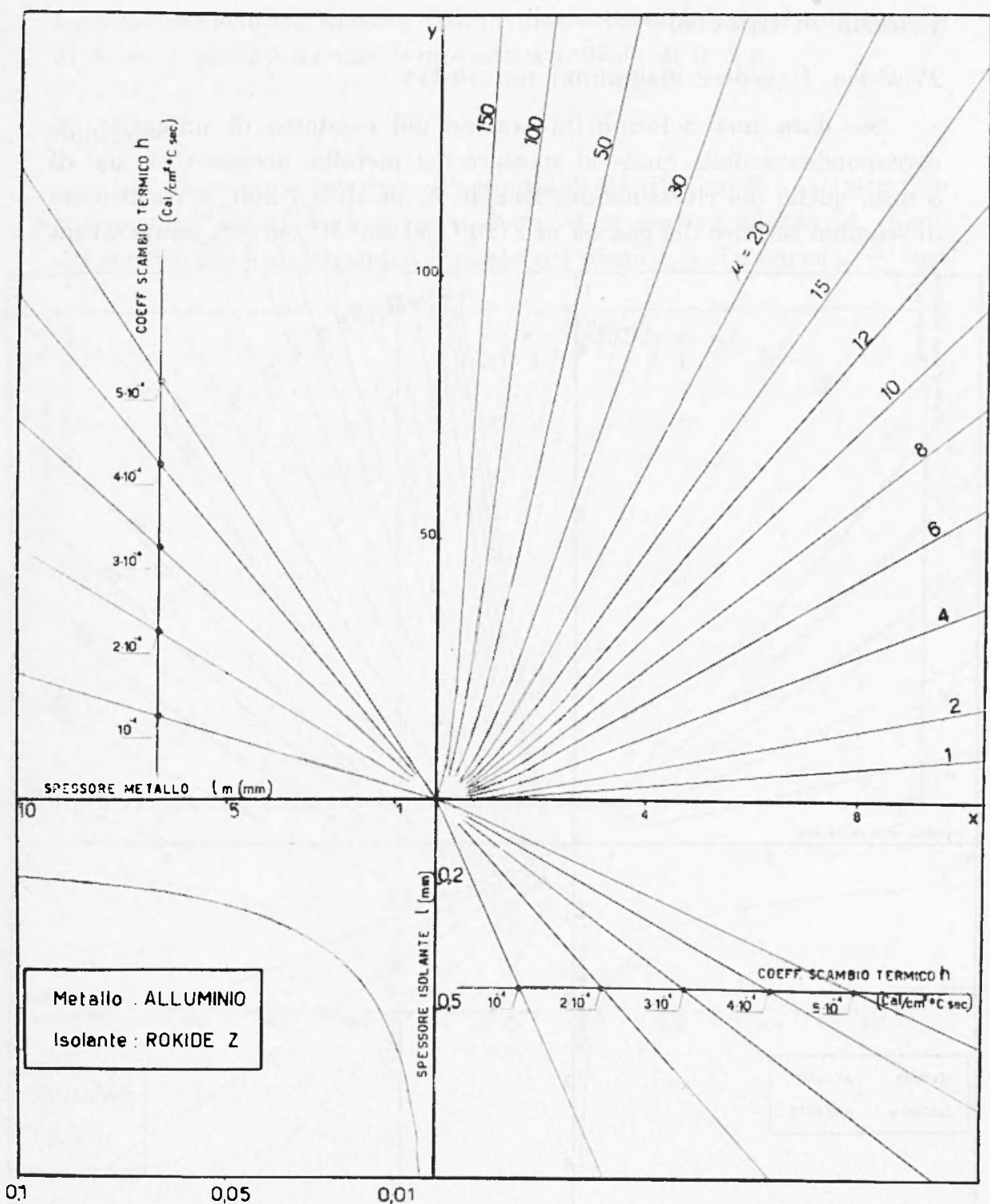

$\alpha\left(\sec ^{4}\right)$

Diagramma $n^{0} 7$-Sistema per la determinazione dei parametri $\alpha$ e $\mu$.. 
Esempio di chicolo.

Problema 1 (vedere diagrammi nn. 10-11).

Sia data ma determinata sezione del condotto di un'ugello, in corrispondenza della quale lo spessore del metallo, acciaio C20 sia di $3 \mathrm{~mm}$, quello del rivestimento, Rokide $Z$, sia di $0,5 \mathrm{~mm}$, il coefficiente di scambio termico dei gas sia di $2 \cdot 10^{-1} \mathrm{Cal} / \mathrm{em}^{2}$ oC sec e la temperatura

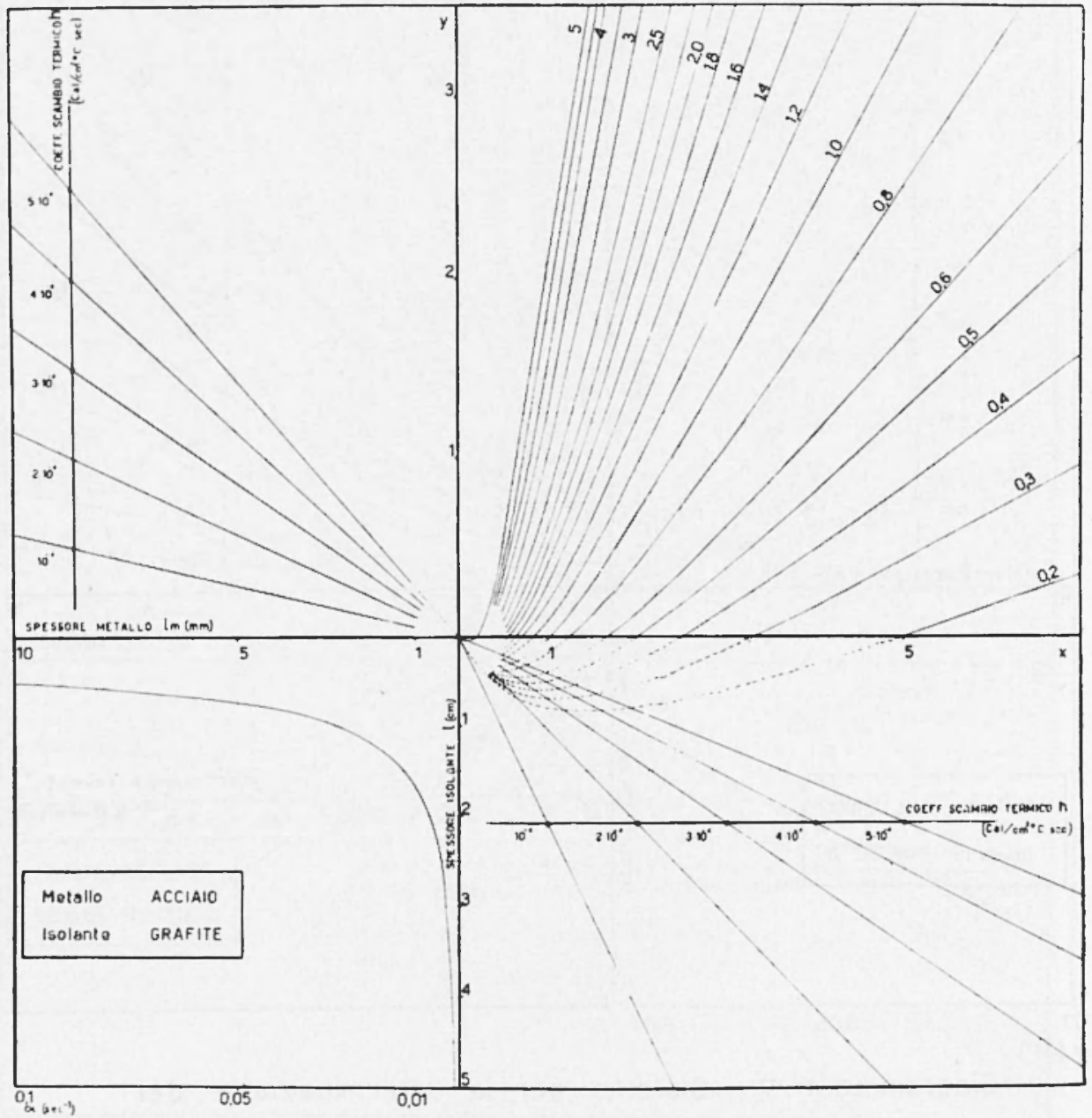

Diagramma $n^{0} 8$ - Sistema per la determinazione dei parametri $\alpha$ e p. - 
CRITERI DI CALCOLO PEI IL RIYESTIMIEXTO TERMICO DI TGELLI, ECC. 283

dei gas in corrispondenza abbia il ralore di $2.000^{\circ} \mathrm{C}$. Si ruole rleterminare la temperatura assunta dall'involucro metallico dopo una rlurata ri 3 sec a partire ra una temperatura iniziale di $0^{\circ} \mathrm{C}$.

\section{Soluzione.}

Fel diagramma relativo allaccoggiamento Rokirle Z - Acciaio C20 si lexgono i valori rlegli spessori detti ed in rorrispondenza al valore rel coefficiente $h$ si rletermina il punto nel piano $x, y$. La curva $\mu=$ cost

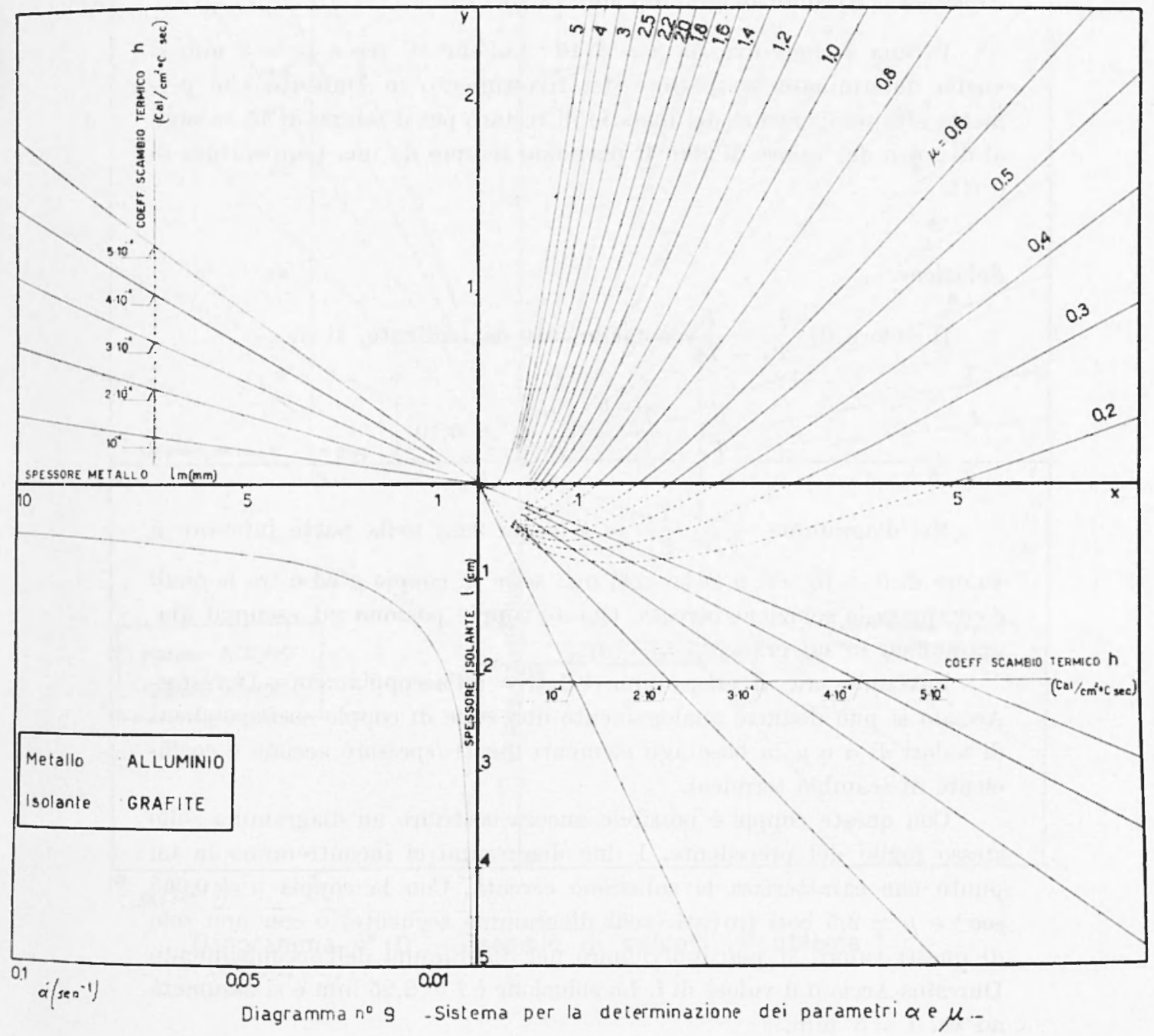


che passa per detto punto è la $\mu=9$ (avendo interpolato tra i ralori di $\mu=8$ e $\mu=10)$. Contemporaneamente si legge in basso il ralore di $a=1,25 \sec ^{1}$. Passando ora nel diagramma $T_{m}-T_{o} / T_{o}-T_{o}=$ $F(\theta)$ si legge il valore di $T_{m}-T_{o} / T_{g}-T_{o}$ corrispondente ai valori $\theta$. a e $\mu$ ciò̀ il valore $\frac{T_{m}-T_{n}}{\bar{T}_{g}-T_{0}}=0,3$ La temperatura del metallo sarà allora

$$
T_{m}=T_{\mathrm{o}}+0,3\left(T_{g}-T^{\mathrm{a}}\right)=0,3 T_{g}=600 \circ
$$

Problema : (vedere diagrammi nu. 12-13-1t)

In una sezione ove sia $h=4 \cdot 10^{-4} \mathrm{Cal} / \mathrm{cm}^{2}$ oO sec e $l_{m}=3 \mathrm{~mm}$ si roglia determinare lo sjessore del rivestimento in Durestos che permetta alla temperatura del metallo di restare per il tempo di 15 secondi al di sotto del valore di 200 oC partendo semple da una temperatura di 0 oc.

\section{Soluzione.}

Il valore di $\frac{T_{m}-T_{0}}{T_{g}-T_{n}}$ è facilmente determinato, si ha

$$
\frac{T_{m}-T_{o}}{T_{g}-T_{o}}=\frac{200}{2000}=0.10
$$

Sel diagramma $\frac{T_{m}-T_{o}}{\bar{T}_{g}-T_{o}}=F(\theta)$, si fissa nella parte inferiore il ralore di $\theta=15$ sece. e si ha cosi ma serie di coppie $\mu$ exh a tra le quali è compresa la soluzione cereata. Queste copple possono ad esempio diaglammarsi in un grafico $\ell=\alpha(\mu)$.

Passando ora al diaglamma relativo all'aceoppiamento DurestosAcciaio si può dedurre analogamente una serie di copple corrispondenti di valori di a e $\mu$ in base agli elementi fissati (spessore acciaio e coefficiente di scambio termicos).

Con queste copple ì possibile ancora costruire un diagramma sullo stesso foglio del precerdente. I due diagrammi ei incontremano in un punto rhe alatterizza la soluzione cercata. Con la roppla $a=0,065$ $\sec ^{1}$ e $\mu \simeq 2,5$ così trovati (vedi diagramma segunente) o con uno solo di questi valori, si può individuare nel diagramma dell'alceopplamento Tourestos-Aceiaio il valore di 7 . Ja soluzione è $l=2,25 \mathrm{~mm}$ a si assumerà ad es. $l=3 \mathrm{~mm}$. 
CRITERI DI CALCOLO PER IT, RIVESTTMENTO TERMICO DI UGELIA, ECC. 285

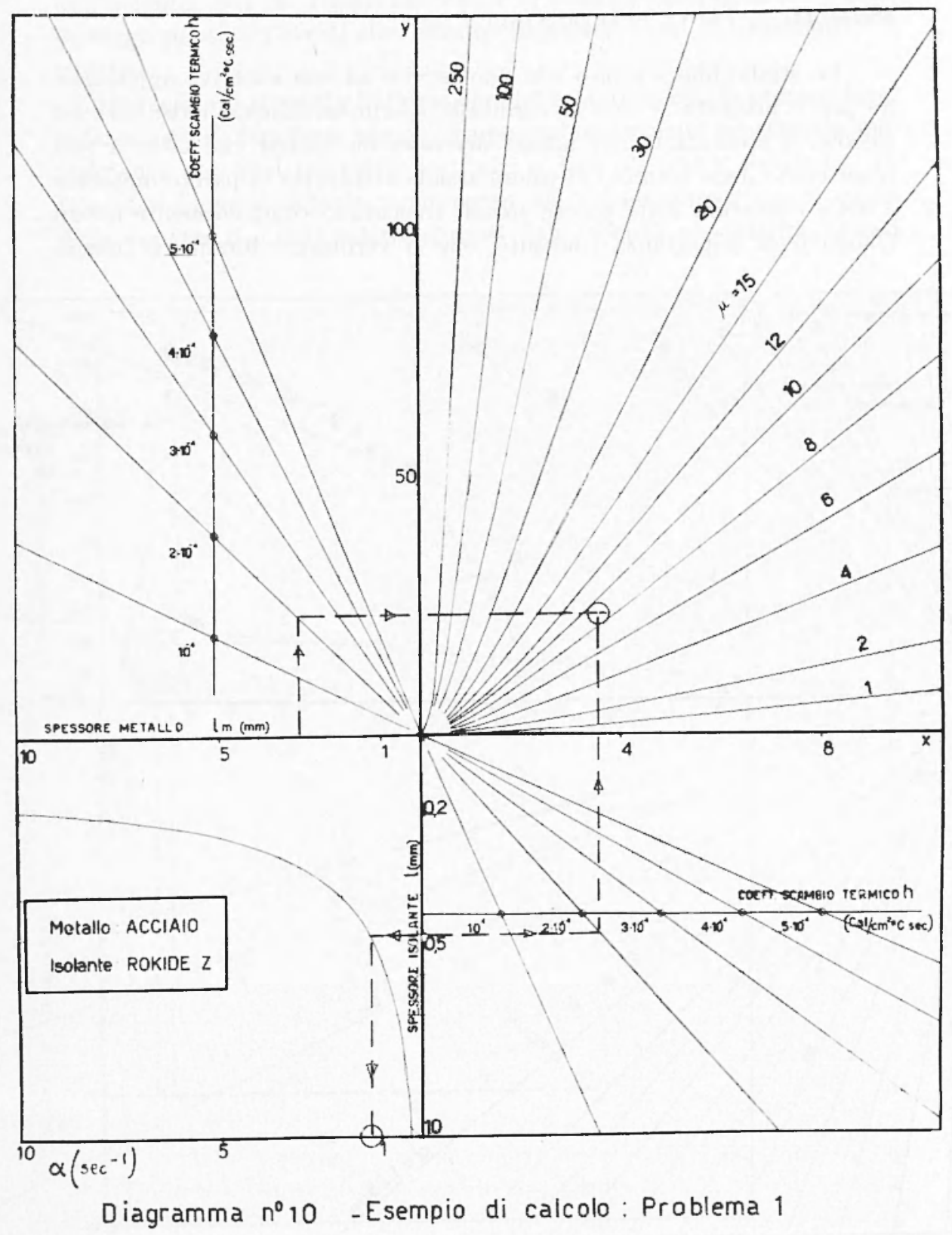


Parte III - PRovf sperimfictil.

Lo studio finora svolto non puó servire ad una elfettiva applicazione per il progetto se non si rerilicano sperimentalmente i risultati del calcolo. I risultati teorici infatti derivano da ipotesi che oltre a non tener conto dello scambio di calore assiale attraverso la parete metallica e della curvatura della parete stessa, trascurano completamente alcuni fenomeni di importanza rilevante, che si verifeano clurante il funzio-

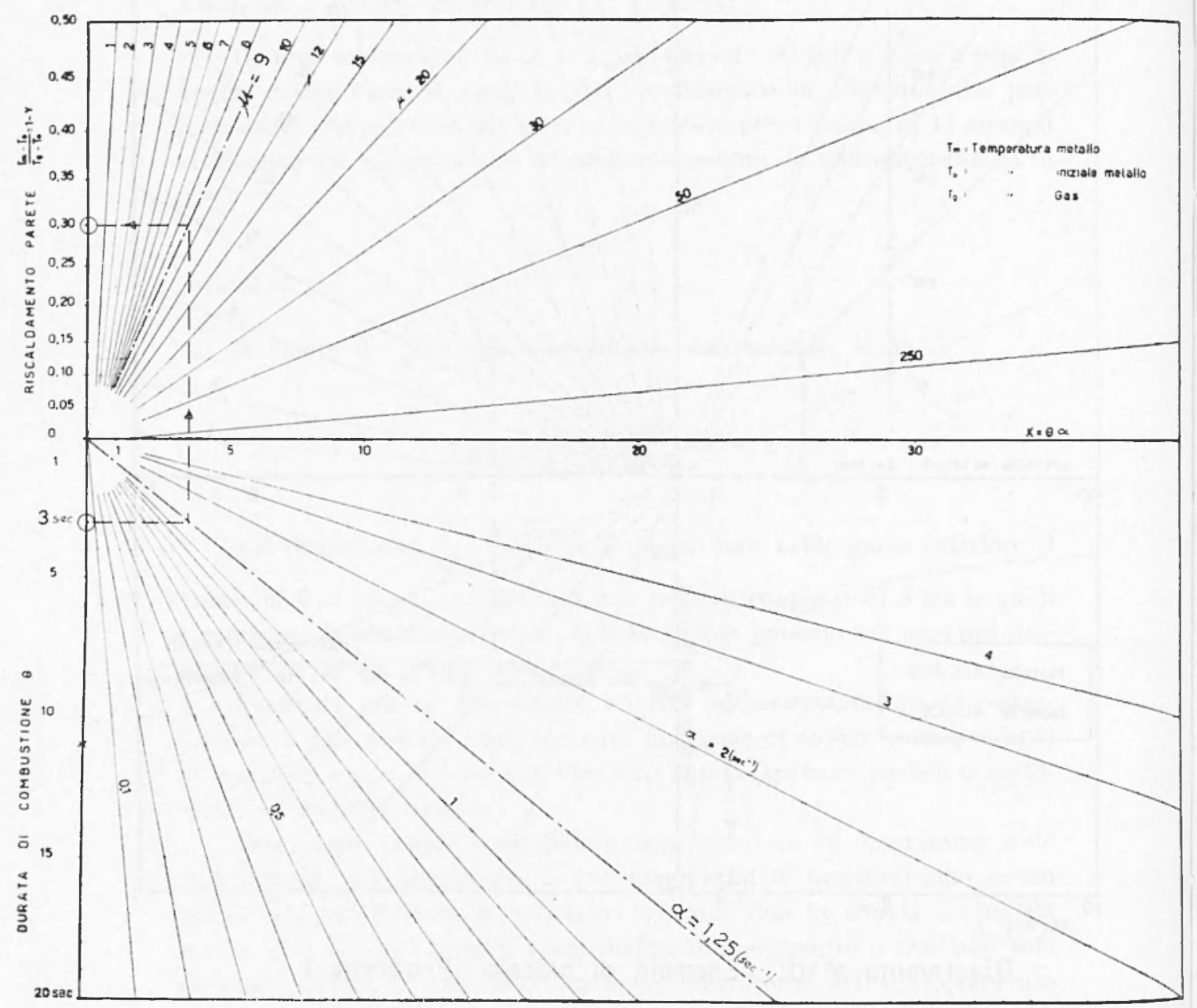

Diagramma $n^{\circ} 11$-Esempio di calcolo : Problema ? 
namento, e ('ioè, ad esempio, la variazione delle caratteristiche dell'isolante con il riscaldamento e l'erosione dell'isolante stesso, l'irraggiamento, leventuale effetto ablativo, con cambiamenti di stato ece.

Per quanto riguarda l'alterazione del materiale con la temperatura, il fenomeno si manifesta spesso in ma carbonizzazione superficiale spe(ialmente per quei materiali costituiti a base di resine sintetiche. Di tale alterazione è difficile tener conto nei calcoli soprattutto a calusa della penuria dei dati relativi forniti dalle societa produturei. Questi

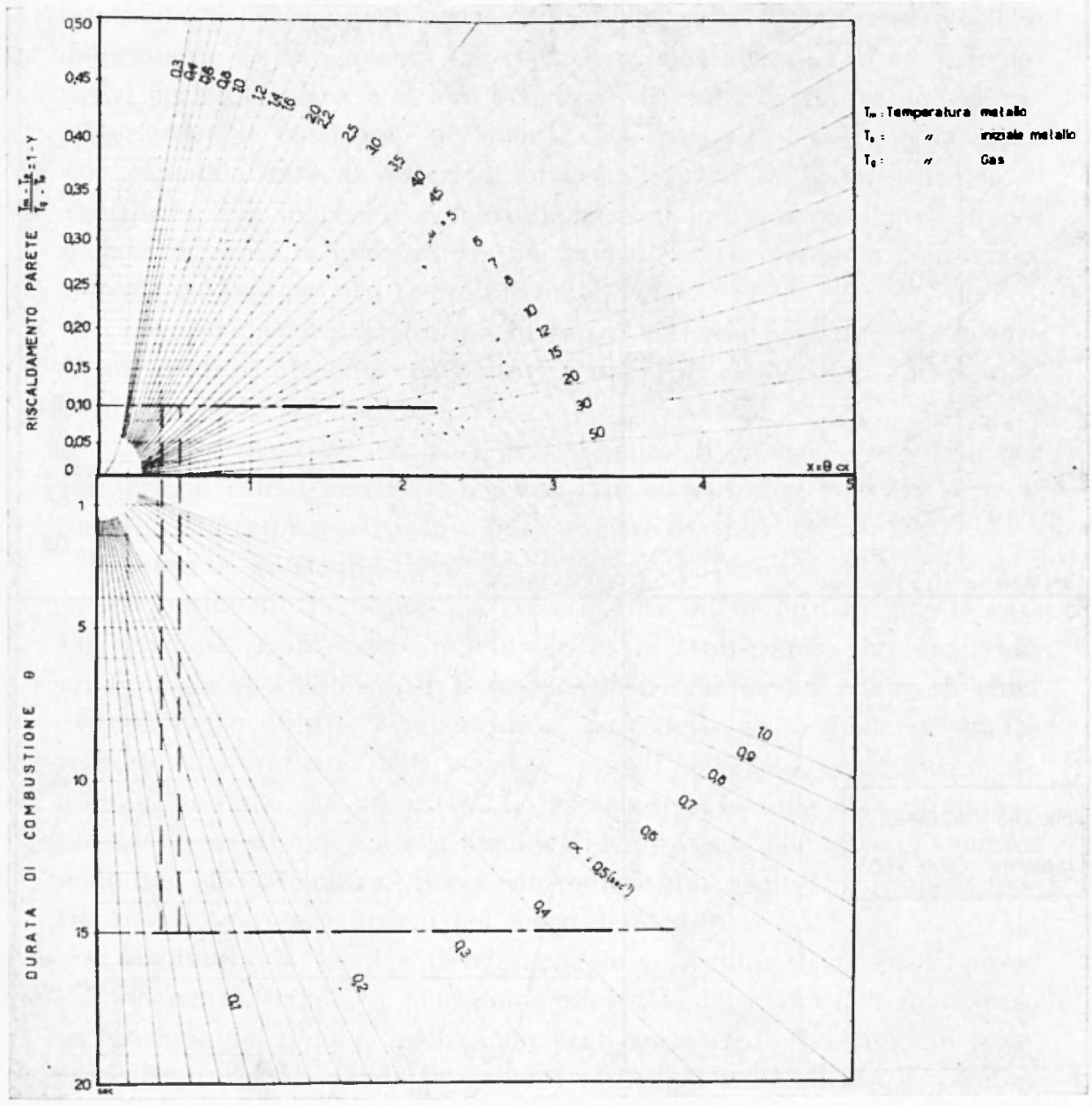

DLagramma n. 12 - Esempio di calcolo: Prohlena 2. 
infatti sono limitati ad intervalli di temperatura eccessivamente ristretti rispetto a quelli di interesse pratico.

L'erosione è un fenomeno notevolmente più complesso e tale, da annullare a volte l'effetto del rivestimento.

Va tuttavia notato che gli effetti di detta erosione sono limitati, almeno nel loro aspetto più pericoloso, alle sezioni del divergente dell'ugello.

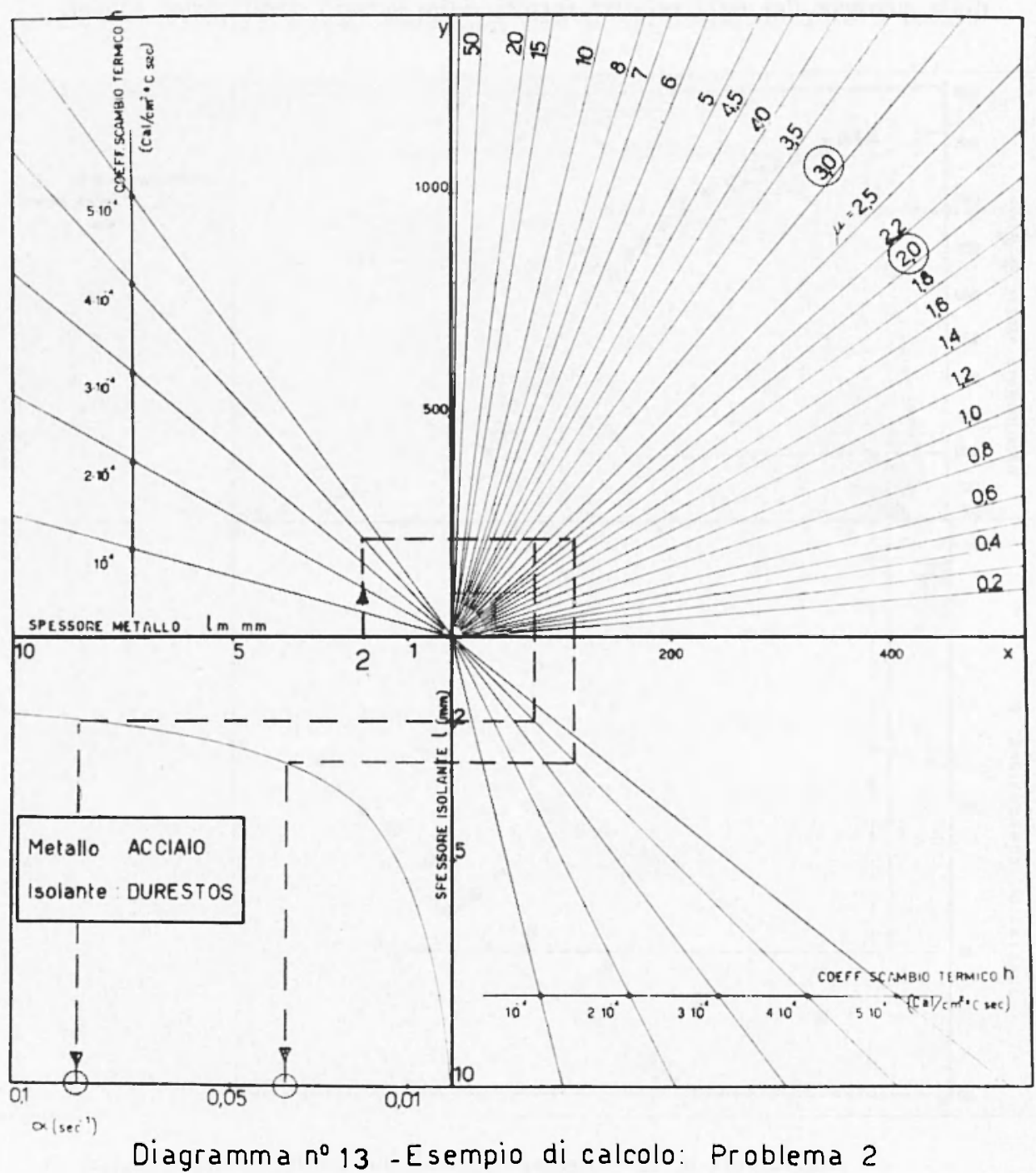


L'erosione inoltre si manifesta soprattutto per quei materiali isolanti a base di resine, cioè per gli isolanti stampabili (Fiberite, Durestos eec.) che sono applicati al supporto mediante incollaggio, mentre è avvertita in misura noterolmente minore nei rivestimenti a base di ossidi metallici, come ad es. $\mathrm{Zr} \mathrm{O}_{2}, \mathrm{Al}_{2} \mathrm{O}_{3}$ che vamno commercialmente sotto il nome di Rokide e (rhe sono applicati al supporto metallico secondo la teconica della proiezione allo stato fuso.

E in corso attualmente uno studio per procedere ad una indagine sperimentale sui vari materiali offerti dal mereato per seguire il loro comportamento in esereizio a varie condizioni, onde definire entro quali limiti questi si accordono con i risultati del calcolo. Salrà così possibile determinare di conseguenza dei coeffieienti sperimentali, ad es. maggiorativi per lo spessore, e la loro eventuale dipendenza dai fattori che caratterizzano le rondizioni di lavoro. Con questi elementi sperimentali sarà possibile dare ai criteri di progetto esposti un valore pratico, e classificare tali materiali isolanti in base a loro campi d"impiego per quanto riguarda le temperature dei propellenti, le pressioni d'esercizio, le parti del motore ed i tempi di combustione.

Le prove sono attualmente in corso; tuttavia possiamo gia formine alcuni risultati oftenuti con motori a tempi di combustione brevi, delloordine cioè di $2 \div 2,5$ sec.

La foto seguente mostra il rivestimento di un ugello oftenuto per stampagugio con il materiale isolante che va commercialmente softo il nome di Durestos, costituito a base di fibre di amianto.

Durante la combustione in vicinanza dell'inserto di guafite si è aruta localmente l'erosione del rivestimento ed miformemente la carbonizzazione. Dalla parte dellinvolucero la temperatuma ha lagegiunto un limite sconsigliabile per il materiale in esame, del valore di circa $300 \mathrm{oC}$, detto "blister temperature ", per effetto della quale sul materiale stesso si formano delle resciche, visibili nella foto, ron conseguente alterazione delle sue proprietà. Lo spessore usato in questa prima ap-

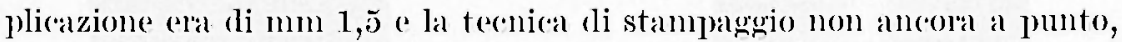
oncle non si è ritenuto di poter confrontare tali risultati con quelli teorici per ricavarne deduzioni nel senso desiderato.

Migliomata la tecnica di stampaggio a seguito di ulteriori prove sullo stesso materiale, si somo infine effettuati dei rilievi di temperature in funzione del tempo, realizzando degli spessori di rivestimento magyiori $(3 \mathrm{~mm}$ ) e bruciando propellenti a diversa temperatura di combustione. Il rivestimento è stato usato sia nel solo converegente che nel divergente. 
F. BOSMAN
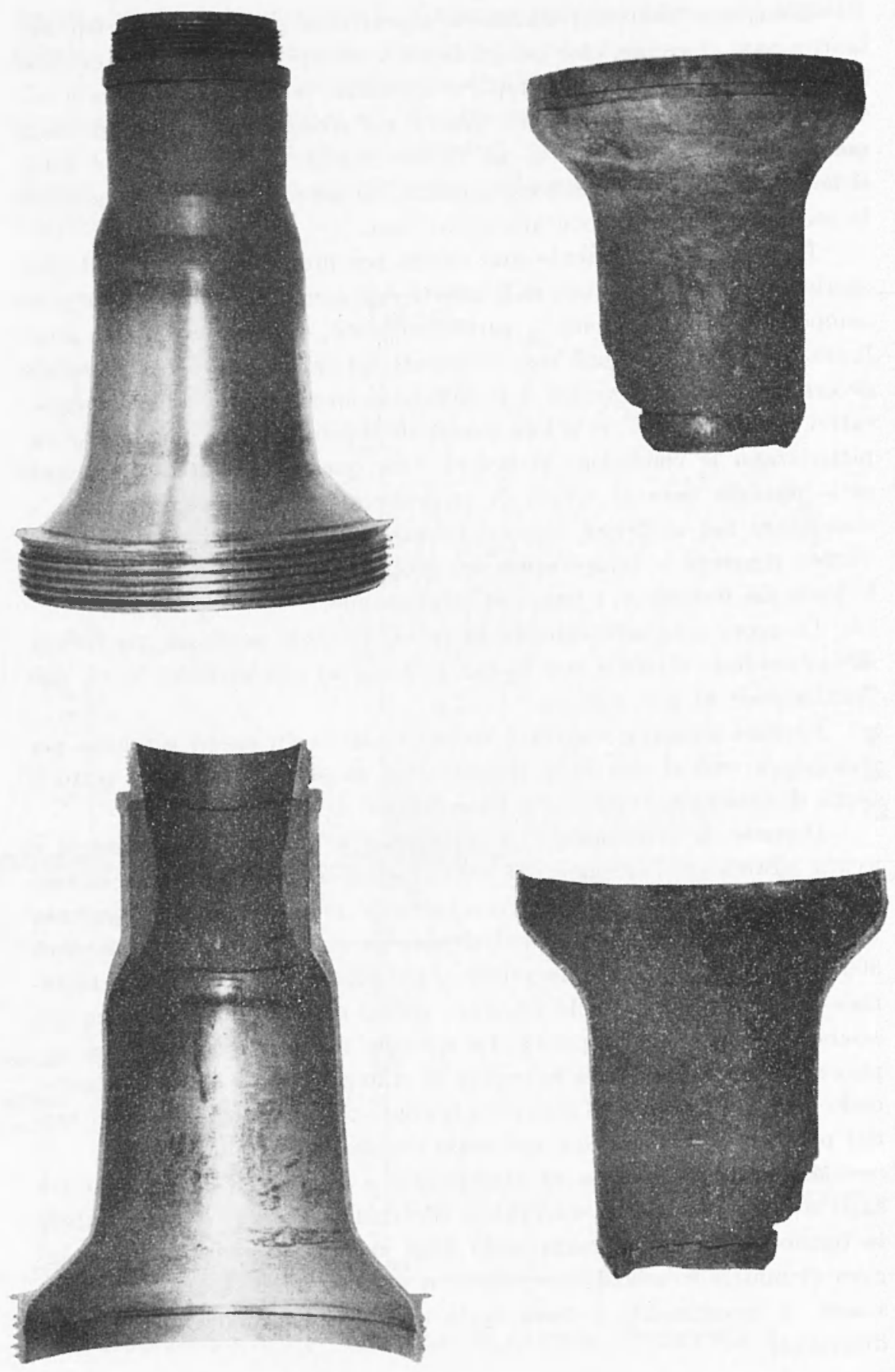
Le misure eseguite con coppic termo-elettriche saldate esternamente allinvolucero metallico hammo dimostrato il non arvenuto riscaldamento nel convergente per tutto il tempo di combustione, in acrordo con i calcoli teorici; nel divergente invece si è avuto un riscaldamento irregolare da attribuirsi molto probabilmente al cedimento del rivestimento; questo infatti durante la combustione es stato completamente asportato dopo una prinal erosione.

Si riportano nei diagrammi segurenti alcuni dei rilievi effettuati.

Il calcolo teorico di confronto precorlentemente effettuato ha richiesto la determinazionc del coefficiente di scambio termico dei gas.

La sua valutazione è stata effettuata applicando l'espressione fornita per tale coefficiente da D. R. Bartz nella pubblicazione: "A Simple

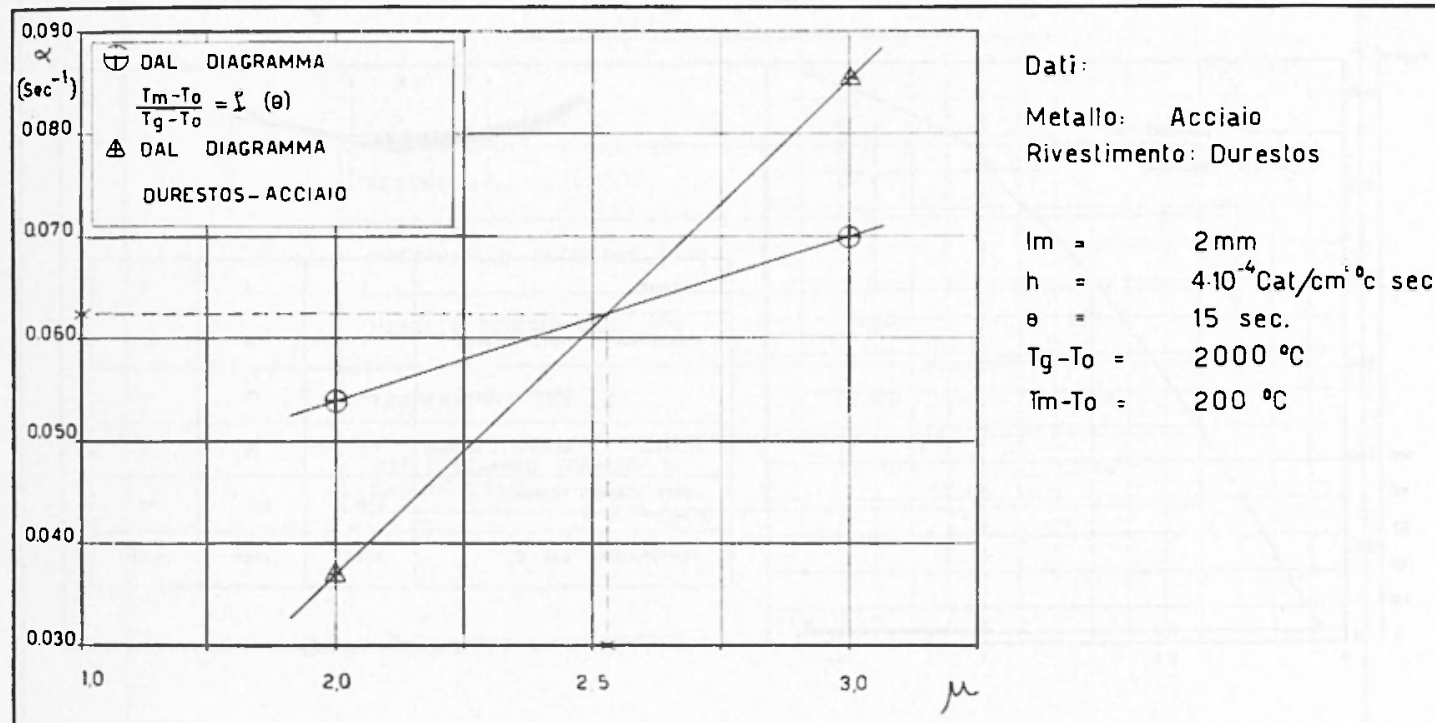

Diag.N.14 Determinazione dei valori di $\alpha$ e $\mu$ relativi ai dali riportati:Problema 2

Equation for Rapid Estimation of Nozzle Convective Heat Tramsfer Coeflicients m. Vedi Jet Propulsion Vol. 27, $1^{\circ}$ 1, Gennaio 1957 ore si tiene conto della sola comvenzione

$$
h=\frac{0,026}{D^{* 0,2}}\left(\frac{\mu^{0,2} C_{p}}{P_{r}^{0,6}}\right)_{0}\left(\frac{P_{c} g}{C^{*}}\right)^{0,3}\left(\frac{D^{*}}{r_{c}}\right)^{0,1}\left(\frac{A^{*}}{A}\right)^{0,9} \sigma
$$




$$
\text { ron } \sigma=\frac{1}{\left.\left[\frac{1}{2} \frac{T_{w}}{T_{o}}\left(1+\frac{\gamma-1}{2} M^{2}\right)+\frac{1}{2}\right]^{0,8(\omega / 5)} \mid 1+\frac{\gamma-1}{2} M^{2}\right]^{\omega(5)}}
$$

$$
\text { ore } \begin{aligned}
A^{*} & =\text { Sezione contratta } \\
A & =\text { Sezione generica } \\
C & =\text { Calore specifico a pressione costante (BTU/lb of }) \\
C & =\text { Telocita caratteristiche (F.P.S) } \\
D & =\text { Diametro della sezione generica (in) } \\
D_{*} & =\text { Diametro della sezione contratta (in) }
\end{aligned}
$$

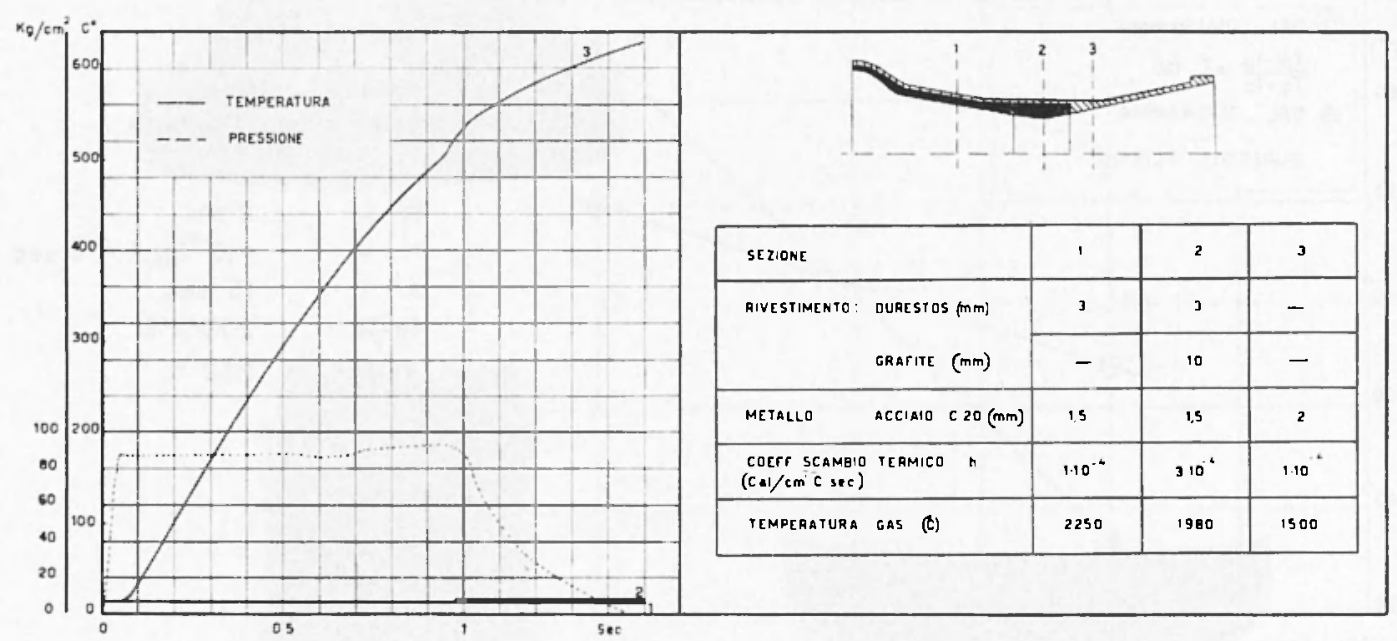

Diag.N" 15 RILICVO SPLRIMEMTALL

$y=$ accelerazione di mavità (F.P.S $\left.\mathbf{S}^{2}\right)$

$h=$ coefficiente di scambio termico dei gas (BTU/in² sec $\left.{ }^{\circ} \mathrm{F}\right)$

$P_{c}=$ pressione nella camera $\left(16 / \mathrm{in}^{2}\right)$

$T_{u}=$ temperatura parete $\left({ }^{\circ} \mathrm{R}\right)$

$T_{o}=$ temperatuma di ristagno $\left({ }^{\circ} \mathrm{R}\right)$ 
CRITERI LI CAJCOLO PER IL RIVESTIMENTO TERMICO DI LGEI,I, ECC. 293

$r_{c}=$ ragoio di curvatura della gola

$n=$ numero di Mach

$\mu=$ viscosità $\left(\mathrm{Ib} / \mathrm{ins} \mathrm{sec}^{\mathrm{e}} \mathrm{c}\right)$

$\gamma \quad=$ rapporto dei calori specifici (schimensionale)

(') = esponente della temperatura nella equazione della viscosità.

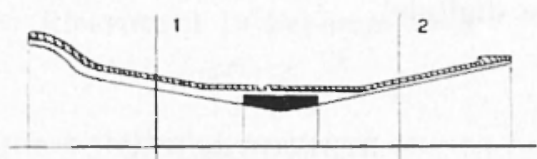

\begin{tabular}{|l|c|c|}
\hline SEZIONE & 1 & 2 \\
\hline RIVESTIMENTO OURESTOS (mm) & 3 & 1.5 \\
\hline METALLO ACCIAIO C $20(\mathrm{~mm})$ & 1.50 & 1.35 \\
\hline TEMPERATURA GAS (C) & 3500 & 2300 \\
\hline $\begin{array}{l}\text { COEFF. SCAMBIO TERMICO } \mathrm{h} \\
\text { (CaV } \mathrm{cm}^{\circ}{ }^{\circ} \mathrm{C} \text { SEC) }\end{array}$ & $1.5 \cdot 10^{-4}$ & $1.5 \cdot 10^{-4}$ \\
\hline
\end{tabular}

Diag. N'16 RILIEVO SPERIMENTALE

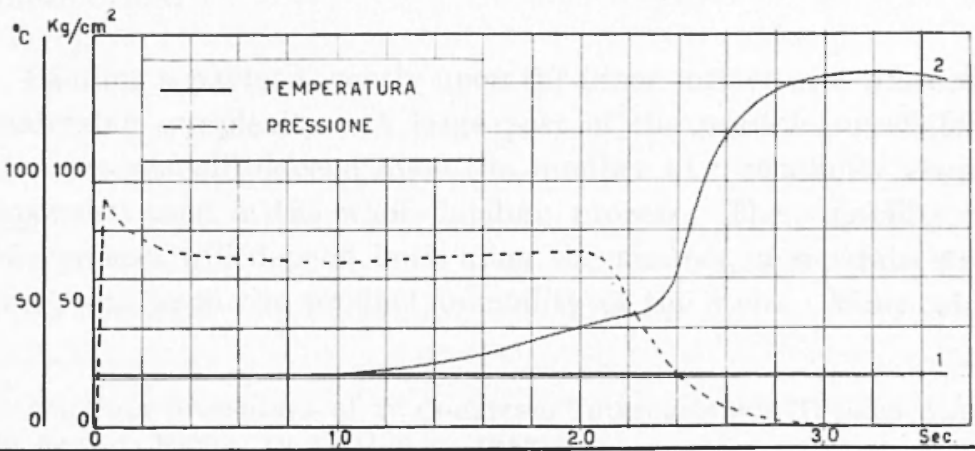


Per quanto riguarda la temperatura dei gias questa si i valutata per le sezioni in esame con la $T=T^{0 / 1}+\frac{\gamma-1}{2} V^{2}$ ponemdo in $T^{0}$ la temperatura di combustione del propellente e valutando $M$ in base al ripporio $\frac{A}{A^{\circ}}$.

Ringrazio il Centro di Calcolo UCC per la fattiva collaborazione prestata ed in particolare il Dott. Giampiero Vergelli per la prestazione alle macedine calcolatrici, Dommer 3400 (calcolat rice analogica) ed IBDL 1620 (computatore digitale).

\section{BIBLIOGRAFIA}

(1) Maxen E., Heat Flow in Composite Slabs. "A Rs-Journal ", 22, (3 Maggio/ Giugno, 1952).

${ }^{(2)}$ BarTz D. R., A Stimple Equation for Rapid Estimation of Rocket Nozzle Convective Heat Transfer Coefficients. "ARS-Journal ", 27, 1, (Gennaio 1957).

$\left.{ }^{3}\right)$ Grover J. H. e Hotter W., Solution of the Transient Heat-Conduction Equation for an Insulated, Infmite Metal. "ARS-Journal", 27, (12 Dicembre 195i). 